\title{
Design of the Lyophilization Process of a Doxorubicin Formulation Based on Thermal Properties
}

\author{
A. A. HAJARE* AND H. N. MORE \\ Department of Pharmaceutical Technology, Bharati Vidyapeeth College of Pharmacy, Kolhapur-416 013, India
}

Hajare and More: Doxorubicin Lyophilization Process Design Using Thermal Properties

\begin{abstract}
The frontline chemotherapy used for treatment of several forms of cancer is doxorubicin, which is required to be lyophilized due to its limited storage stability. Results from differential thermal analysis/ impedance and freeze drying microscopy determinations, signifies that the freezing step product temperature must be well below $-\mathbf{5 9 . 8}^{\circ}$, while throughout the primary drying stage product temperature at sublimation front cannot exceed $-19.7^{\circ}$. The use of a procedure based on the critical parameters allowed freeze drying of doxorubicin laboratory scale batches that would help to reduce process cycle time, energy and costs at commercial production.
\end{abstract}

Key words: Doxorubicin, lyophilization, cycle design, sublimation, electrothermal analysis, freeze drying microscopy

Doxorubicin hydrochloride (DOX) is an antineoplastic drug effective against lymphoma, carcinoma and various sarcomas ${ }^{[1]}$. It is very stable at room temperature in the solid state and has been found to be unstable in solutions with loss in potency. DOX degradation has been reported when stored for three months at $60^{\circ[2]}$. Various types of DOX formulations are available commercially but pharmaceutical problems make it complicate to use. Slow and incomplete dissolution upon reconstitution make some of these formulations unsuitable to be used with safety. DOX is inactivated via various pathways ${ }^{[3-5]}$. Shelf life of DOX can be enhanced by lyophilisation by several folds. The lyophilized DOX is reconstituted with solvent and bulk dosages are stored for further use. The reconstituted solutions are stable only for a period of $48 \mathrm{~h}$. However, it is recommended that the solution be stored at $2-8^{\circ}$ in a refrigerator, and used within $24 \mathrm{~h}$.

Lyophilization is a freeze-drying operation used for long-term stabilization of actives degrades in water and sensitive to higher temperatures. The benefits of the process include extended shelf life and cheaper storage and transportation costs relative to the liquid form of the product ${ }^{[6]}$. It is comprised of freezing, sublimation (primary drying), and desorption (secondary drying). Lyophilization stabilizes the drug in formulation by slowing down the degradation process. It modifies the nature of materials by eliminating the solvent

*Address for correspondence

E-mail: ashok.hajare@bharatividyapeeth.edu

November-December 2017

Indian Journal of Pharmaceutical Sciences component to level that it no longer supports chemical changes to occur. Solvent removal is accomplished by freezing the formulation and immobilizing any solvent in the interstitial region between the crystals. The crystal microstructure formed during freezing determines the final quality of the material and lyophilizate structure. Ice is further eliminated by sublimation during primary drying and is continued until all of the ice surrounding the interstitial space is removed ${ }^{[7]}$. This step requires decrease of the chamber pressure below the ice vapour pressure in the sublimation front. The bound water remaining in the structure at the end of the sublimation step $(\approx 10 \%)$ is eliminated in the secondary drying step. This last step is performed at higher temperatures to guarantee that water is desorbed through diffusion from the structure ${ }^{[8,9]}$.

High development and manufacturing costs has made this process invariably reserved for 'high value' products ${ }^{[10]}$. Quality by design approach might significantly reduce both the product development time/cost as well as subsequent operational costs

This is an open access article distributed under the terms of the Creative Commons Attribution-NonCommercial-ShareAlike 3.0 License, which allows others to remix, tweak, and build upon the work non-commercially, as long as the author is credited and the new creations are licensed under the identical terms

Accepted 16 September 2017

Revised 19 March 2017

Received 30 July 2016

Indian J Pharm Sci 2017;79(6):907-913 
through a more rational design of the product and an optimised freeze-drying process. Shortening the process duration without compromising the quality of the final product is one of the major challenges during lyophilization ${ }^{[11-13]}$. Freeze-drying cycle design is mainly based on process and formulation variables. The determination of glass transition temperature helps to set freezing temperature of the product, collapse temperature for formulation determines the operational parameters during sublimation and the proper product characterization facilitates the execution of a safe and efficient process ${ }^{[14,15]}$. A variety of analytical techniques were available to estimate the heat and mass transfer rates in the drying chamber and condenser to achieve such characterization ${ }^{[16,17]}$. These techniques include gravimetric measurements, product temperature sensors and heat transducers, manometric temperature measurements, tuneable diode laser absorption spectroscopy, visual monitoring of the ice interface, differential scanning calorimetry (DSC), differential thermal analysis (DTA) and electrical impedance (EI), and freeze drying microscopy (FDM). DSC and DTA take advantage of any physical change occurring in the structure of the lyophilized compound related to either an exo- or endothermic phenomenon. EI determinations (Zsin $\varphi$; $\mathrm{kOhms}$ ) provide evidences on matrix mobility relative to temperature. FDM allows the real time observation of the matrix at microscopic level during the entire process and thus regarded as the genuine technique to determine the collapse temperature of the structure. Although these techniques have advantages and limitations, their combination is essential to determine the critical temperature values that guarantee the quality of the lyophilizate obtained to achieve reliable lyophilization rates ${ }^{[18,19]}$.

The present research work was aimed to design the lyophilization process of the DOX. This article provides a brief insight on the applications of DTA, EI and FDM technology in the characterization of the lyophilization process, using a placebo formulation containing lactose as cryoprotectants and DOX formulation along with components of placebo formulation in distilled water. The main focus of this article is determination of the end points of the freezing and the primary drying process.

\section{MATERIALS AND METHODS}

The following materials were used in the formulation: DOX hydrochloride (Intas, India), lactose (HiMedia, Mumbai), polyvinyl pyrrolidone K30 (Sisco, India) and sodium dihydrogen phosphate (Merck, India). Bottling materials were clear natrium-calcium tubular $5 \mathrm{ml}$ glass vials (Yantai Uech Pharmaceutical Package Co., Ltd, China) and dry vented butyl rubber closures (Apipharma, India). Materials were lyophilized in a Heto LyoPro 3000 (Heto-Holten A/S, Allerod, Denmark) with collector temperature $-50^{\circ}$, ice holding capacity 5 , refrigerant HFC type R507, analogue controller with 'Alarm-Wait-Okay' status indicator and temperature read-out.

\section{Electrothermal analysis (DTA/Zsin $\varphi)$ :}

The following materials were used in the formulations for electrothermal analysis. Placebo formulation: lactose $100 \mathrm{mg} / \mathrm{ml}$, polyvinyl pyrrolidone $\mathrm{K} 30$ $3 \mathrm{mg} / \mathrm{ml}$ and sodium dihydrogen phosphate $15 \mathrm{mg} / \mathrm{ml}$. Drug formulation: DOX hydrochloride $4 \mathrm{mg} / \mathrm{ml}$ and lactose $100 \mathrm{mg} / \mathrm{ml}$, polyvinyl pyrrolidone $\mathrm{K} 303 \mathrm{mg} / \mathrm{ml}$ and sodium dihydrogen phosphate $15 \mathrm{mg} / \mathrm{ml}$.

Lyotherm 2 has been designed to measure critical temperatures of freeze-drying formulations. The use of impedance analysis for the measurement of a derivative of impedance known as "Zsin $\varphi$ " or " $\mathrm{k} \Omega$ " provides information about behaviour of the frozen solute phase, which in most cases is not identifiable using conventional analytical techniques ${ }^{[20]}$. Temperature (DTA) and impedance (Zsin $\varphi)$ analyses was performed in a Lyotherm2 equipment (Biopharma Technology Ltd, Surrey, UK), consisting of Lyotherm2 as main component of the system. DTA and Zsin $\varphi$ sensors were immersed in the cuvette containing the samples (DOX formulation) and the reference substance (distilled water), kept in cooling/heating block. In this $6 \mu 1$ sample was filled in each of the sample holders. One of the samples was used for recording differential thermogram and another for recording EI. One probe was kept in reference holder, which contained distilled water and second probe kept in block. Cooling was achieved by filling liquid nitrogen in reservoir tank. Upon filling the sample and reference solutions the block was kept in reservoir tank and the recording was started using the Lyotherm 2 software. The cooling started up to $-90^{\circ}$, followed by block pulling upward in tank and the heating was started at $2 \% \mathrm{~min}$. When the temperature reached $0^{\circ}$, recording was stopped and the data was taken on excel sheet to plot the graph. The $Z \sin \varphi$ determination was made while heating. Data acquired using Lyotherm2 program was processed using Microsoft Excel 2010 for ensuing analysis. The Lyostat2 graph shows a sharp change in temperature/ impedance indicating sample collapse ${ }^{[20]}$. 


\section{FDM:}

Lyostat2 is a fully integrated FDM that enables to determine product structure collapse and melting in situ, as well as identifies the time and temperature of skin or crust formation ${ }^{[20]}$. The system comprises of a polarizing microscope and a vacuum tight temperature controlled stage called micro-freeze dryer. To investigate the freeze dried behaviour of a placebo formulation and the effect of DOX incorporation, $2 \mu \mathrm{l}$ placebo formulation containing lactose, sodium dihydrogen phosphate and polyvinyl pyrrolidone K30.

The process of FDM began with loading a sample into a stage chamber under the microscope followed by lid closure. The amount of placebo formulation required for Lyostat2 is $2 \mu \mathrm{l}$. Automatic precision cut spacers (brass platelets, height $0.025 \mathrm{~mm}$ ) controlled thickness of the sample layer. Liquid nitrogen used for cooling attains a standard lowest freezing temperature $\left(-60^{\circ}\right)$. The compositions under investigation were frozen to $-60^{\circ}$ between quartz cover slips in the freeze dried stage of Lyostat2, without annealing. The sample was cooled up to $35^{\circ}$ at the freezing rate of $5^{\circ} / \mathrm{min}$. At this temperature, a sample was held for 5 min to purge the system with dry nitrogen from the Dewar through the open valve. Prior to sublimation, sample was held at the lowest freezing temperature to attain equilibrium for $5 \mathrm{~min}$. The vacuum pump was used to dry the sample. The freezing and drying behaviour of sample was determined visually. A controller computer system captures images and related data. Micrographs were recorded using a video camera at $150 \mathrm{x}$ magnification. Throughout the entire cycle pictures were taken with the delay of $10 \mathrm{~s}$ using a digital fire wire camera (resolution 1.3 megapixels) and analysed using the Lyostat2. The pressure is controlled well below 0.02 mBar. The saved pictures were compared using custom-made supporting software. Software supplied with Lyostat2 allows control over the freeze-drying, displaying and recording temperature and vacuum readings.

The investigation of thermal properties of a formulation component was done by determining collapse temperature using Lyostat2. All the above information is useful for screening formulation components if required and for the cycle development.

\section{Methodology for designing the lyophilization process:}

The critical temperatures obtained for placebo formulation from DTA/EI and FDM studies were applied for the freeze drying of DOX formulation. Accurately measured $2 \mathrm{ml}$ solutions were filled in $5 \mathrm{ml}$ glass vials. The vented rubber closures were positioned as half-closed. The solutions were frozen at $-35^{\circ}$ for $4 \mathrm{~h}$ in deep freezer (Polar 530V, Angelantoni Industries India). The pre-frozen vials were freeze dried in Heto LyoPro 3000 at shelf temperature of $-25^{\circ}$ and pressure $0.016 \mathrm{mBar}$, for $96 \mathrm{~h}$ (condenser temperature: $-50^{\circ}$ ). The freezing and sublimation safety temperatures were determined according to the results obtained in characterization studies (DTA/EI and FDM). These temperatures were set at approximately $5^{\circ}$ below the previously determined collapse temperature.

The shelf temperature and the chamber pressure remain steady during sublimation drying. Since equipment used has no manometric temperature measurement facility; the temperature of the shelf was set to a value similar to collapse temperature. This was to assure that the collapse temperature could not be surpassed during sublimation. Chamber pressure was determined by putting values in equation reported in literature ${ }^{[21,22]}$. The calculated value was controlled by injecting dry air into the chamber. Product temperature was substituted by freezing and sublimation safety temperatures, to obtain an optimum pressure to guarantee the safety and efficiency of the process $^{[22]}$.

Secondary drying was performed by heating the shelf at a $0.5^{\circ} / \mathrm{min}$ until reaches $25^{\circ}$ and that value maintained until the temperature of product surpassed $15^{\circ}$. Subsequently, based on results obtained in previous studies of accelerated stability of the DOX, shelf temperature was increased to $35^{\circ}{ }^{[23]}$. No significant changes were detected in the performance of the system under screening. The chamber pressure was set below $0.02 \mathrm{mBar}$ during this step. The freeze dried products were protected from environment, light and moisture. At the end of cycle design, a confirmation run was conducted to ensure that the new cycle produces acceptable product and runs flawlessly. A full dryer ( 3 shelves) loaded with both the DOX and placebo formulations was processed. All the DOX vials were inspected for any physical defects such as collapse, meltback and shrinkage.

\section{Cake appearance:}

The appearance of the freeze dried product cake was inspected visually for its structural integrity and pharmaceutical elegancy.

\section{Residual moisture:}

The residual moisture level in freeze dried products 
was determined by using a Karl-Fischer titrator (VeegoMatic MD). Accurately measured $20 \mathrm{ml}$ anhydrous methanol was transferred to the titration vessel and titrated to the endpoint. Karl Fischer reagent was standardized using $10 \mu \mathrm{l}$ of distilled water. Randomly 3 vials were selected and accurately weighed $5-20 \mathrm{mg}$ samples from each vial were separately suspended in anhydrous methanol and titrated to the electromagnetic end point.

\section{Reconstitution time:}

Reconstitution time of the lyophilized products was obtained using Thiermann method. Each sample was reconstituted with $2 \mathrm{ml}$ water for injection, IP. The vials were shaken horizontally at a distance between 6 inches on hard surface to form the solution. The vials were visually inspected to measure reconstitution time with absence of any visible aggregates.

\section{RESULTS AND DISCUSSION}

FDM and DTA/EI techniques determine collapse temperature quickly and accurately in less than one hour. Performance and efficiency of these methods was compared with conventional methods used to design lyophilization cycle. Generally, product during lyophilization in vial collapses at a slightly higher temperatures. The collapse temperature is solute concentration dependent and the effect is insignificant in systems with low solutes due to rapid sublimation ${ }^{[24]}$. To minimize the variation, the collapse temperature measurements by FDM were conducted with solute concentrations comparable to the concentrations conventionally used. The observed collapse temperature is the highest allowable product temperature during sublimation in primary drying. The lyophilisate structure collapses if the product temperature exceeds highest allowable temperature during this stage.

The impedance of placebo formulation recorded using Lyotherm 2 showed sharp change in curve at $-8^{\circ}$ with complete lyophilisate structure collapse at $-3^{\circ}$. The result of impedance $(Z \sin \varphi)$ determination of the DOX formulation run on Lyotherm2 (fig. 1) shows that as heating progresses a shift in the slope of $\mathrm{Z}$ heating curve (impedance) occurs due to softening event at $\mathrm{T} 1$ $\left(-70.7^{\circ}\right)$, which has no counterpart in DTA and must thus be minimally thermal (or isothermal) in nature. At T2 $\left(-59.8^{\circ}\right)$ there is a sharp decrease in impedance indicates rearrangement of frozen structure. After T3 $\left(-57.0^{\circ}\right)$ impedance decreased consistently indicating structure stabilisation. The negative inflexion at this temperature demonstrates the destabilization of the matrix but in this case it seems to have no influence on lyophilization parameters. There was no recrystallization of any of the DOX formulation components. Since T2 $\left(-57.0^{\circ}\right)$, the temperature steadily increased indicating increased interstitial mobility until the structure collapse is reached at $\mathrm{T} 4$ $\left(-5 \cdot 0^{\circ}\right)$. The minimum impedance at T5 $\left(-1^{\circ}\right)$ indicates fusion of solute structure. Incorporation of DOX in formulation shifted collapse temperature towards zero by $3.0^{\circ}$.

The DTA thermograph of placebo formulation showed endothermic peak at $-8.0^{\circ}$. The DTA thermogram of DOX formulation lyophilizate run on Lyotherm2 (fig. 2) showed that at T1 $\left(-5.0^{\circ}\right)$ starts an endothermic pattern indicating a phenomena related to the destabilization of the frozen crystal structure. The perfectly defined endothermic peak initiated at T2 $\left(-1.0^{\circ}\right)$ indicates the start of matrix structure fusion.

The pictures of placebo formulation captured during FDM (Lyostat2) using FDM indicated no structure collapse up to $-8^{\circ}$. The DOX lyophilizate pictures (fig. 3) captured during FDM studies shows start of structural morphology modifications. Status of collapse temperature determination during freeze drying of the placebo and DOX formulations under

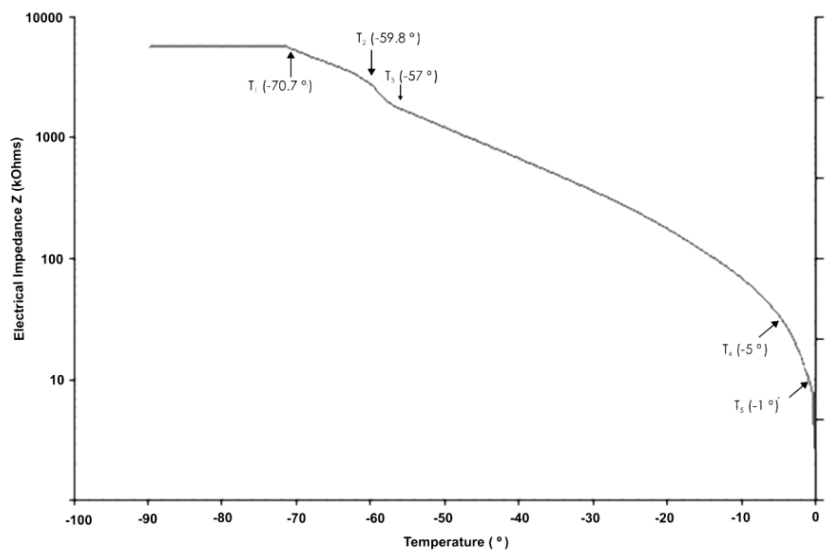

Fig. 1: Impedance $(Z \sin \varphi ; k \Omega)$ of the DOX lyophilizate formulation

Sample was cooled down to temperatures below $-90^{\circ}$ and immediately heated until room temperature at a rate of $2 \% \mathrm{~min}$. T1: matrix destabilization temperature. T2: temperature of complete mobility within solute structure. T3: temperature of ice crystals rearrangement. T4: structure collapse temperature. T5: temperature of ice crystals fusion. Above $-5.0^{\circ}$ there is an increase in downward gradient of impedance curve indicating a softening of the frozen material. At $-\mathbf{1 6 . 0}^{\circ}$ there was an increase in impedance indicating a rearrangement of frozen structure confirmed by Lyostat 2 as complete collapse at $\mathbf{- 1 . 0}$ 
study are given in Table 1 . In the lyostat 2 analysis of both types of formulations drying front progressed at high speed with no product collapse up to $-20.0^{\circ}$, material got completely dried below $-19.7^{\circ}$. This type of phenomenon resulted due to absence of any water mobility in the matrix ${ }^{[25]}$.

The DOX lyophilizate structure collapse was detected

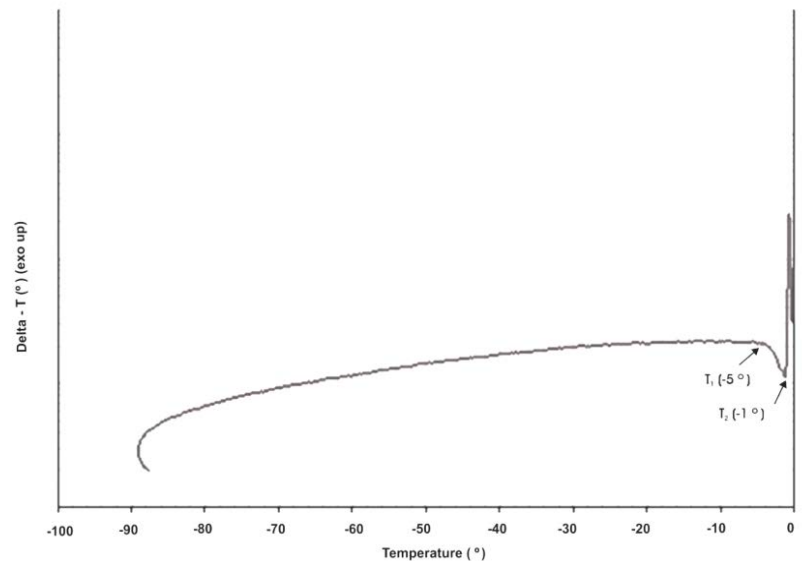

Fig. 2: Differential thermal analysis (DTA) curve of the DOX lyophilizate formulation

Sample was cooled down to $-90^{\circ}$ and subsequently heated until room temperature at a rate of $2 \% \mathrm{~min}$. T1: matrix destabilization temperature. T2: temperature of complete mobility within solute structure. At around $\mathbf{- 7 0 ^ { \circ }}$, there is the beginning of an exothermic event (shown in DTA) that leads to the softening of the sample at $\mathbf{- 5 9 ^ { \circ }}$. Maximum molecular mobility is achieved at $-5^{\circ}$, identified with the start of some melting (increasing downwards slope on the DTA heating curve). Full melt occurs close to $-1^{\circ}$
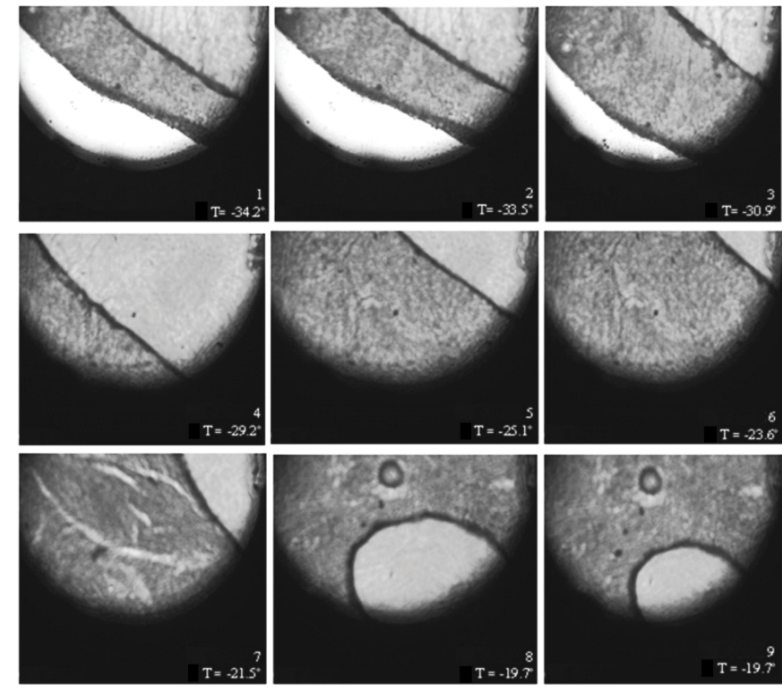

Fig. 3: Determination of collapse temperature (Tc) of the DOX formulate, by LM technique

Sample frozen at $-60.0^{\circ}(2 \% \mathrm{~min})$ followed by heating to $-10^{\circ}$ $(1 \% \mathrm{~min})$ at $0.016 \mathrm{mBar}$ pressure. Picture 1 to 5 shows absence of structure collapse up to $-25.1^{\circ}$. Picture 9 shows completely dried structure at $-19.7^{\circ}$. The composition was essentially sublimed below $-5.0^{\circ}$ at temperatures lower than T3 (fig. 1) and T1 (fig. 2), which is rational because such values correspond to possible destabilization of the frozen material. The $\mathrm{Z}$ heating and DTA heating graphs showed two clear effects that illustrate the synergy between EI and DTA. The glassy transition of the matrix is the temperature at which the frozen material progressively softens because of the increased mobility in the interstitial zone of matrix ${ }^{[7]}$. The glass temperature $\left(\mathrm{T}_{\mathrm{g}}\right)$ value is of the same magnitude, being within the range as reported in the literature in respect to collapse temperature, considering that DTA curve shows the start of an endothermic profile at $\mathrm{T} 1\left(-5.0^{\circ}\right)$ differing in $3^{\circ}$ from collapse temperature $\left(-2.0^{\circ}\right)^{[26,27]}$.

Based on previously discussed placebo and DOX formulation FDM/EI results, the freezing temperature of the product was set to $-30.0^{\circ}$, with a margin of $29.0^{\circ}$ below collapse temperature. The shelf starting temperature was set to $-45.0^{\circ}$, with a decrease in temperature at speed of $1.0^{\circ} / \mathrm{min}$. When the product reached values below $-30.0^{\circ}$, the shelf temperature was increased up to $-35.0^{\circ}$ to stabilize the matrix in the desired temperature range. This was followed by a $1 \mathrm{~h}$ stabilization period prior to begin the sublimation ${ }^{[6,28]}$.

In order to avoid surpassing the previously established collapse temperature value during primary drying the shelf temperature was set to $-25.0^{\circ}$. In reality, Lyostat 2 studies, along this step the temperature of the products remained below the freezing and sublimation safety temperature $\left(-5.0^{\circ}\right)$. The chamber pressure $0.16 \mathrm{mBar}$ was kept within the range $0.14-0.18 \mathrm{mBar}$ by injecting dry air in the chamber. The end of primary drying was determined by detecting the change in the slope of the curve of temperature values, which were close to the set shelf temperature ${ }^{[29]}$.

The secondary drying product temperature was set on the basis of thermal stability data obtained from the initial data reviewed ${ }^{[30]}$. Thus, this final step of lyophilization was carried out at a shelf temperature of $35^{\circ}$. Chamber pressure and condenser temperature were used to determine the end of this process. Once chamber pressure falls down below $0.06 \mathrm{mBar}$ and condenser temperature below $-50^{\circ}$, the chamber pressure change test was performed at $1 \mathrm{~h}$ interval. The process was regarded as complete when the pressure difference was $<0.01 \mathrm{mBar} / \mathrm{min}$.

The information gained about critical parameters associated with the lyophilization cycle design 
indicated the increase of the freezing temperature by $5^{\circ}$, shortening the duration of this step (Table 2). Shelf temperature increased during primary drying reached $-30^{\circ}$ and the chamber pressure to $0.16 \mathrm{mBar}$. The whole duration of the lyophilization cycle (Table 3 ) as designed in dealing to determine formulation critical variables, was of $9.5 \mathrm{~h}, 34.5 \%$ shorter than the duration of the previously established "trial-and-error" method reported earlier ${ }^{[30]}$. New process cycle designed based on optimized critical parameters supports an increase in the production capacity, making able to execute bonus for processes in the same time compare to conventional ones.

DOX formulation lyophilized using the newly established critical parameters complied with quality specifications. The organoleptic irregularities in lyophilized product are associated with alterations during process due to stresses. The physical appearance of the lyophilisate showed red porous cake (pill) of regular edges and structures devoid of cavities or deformations. The porous dried solid dissolved immediately, leading to a clear solution with no solid particles. The length of the secondary drying stage depends on the desired residual moisture content. The percent residual moisture was lower than $3 \%$. The DOX content determined using HPLC was within the established limits.

Presently no other technique than DTA/impedance and real time FDM using Lyotherm2 and Lyostat2 is specifically designed to analyse the liquid frozen state of the freeze drying process. The results of the combination of real time DTA/Zsin $\varphi$ and FDM techniques indicate that incorporation of DOX shift collapse temperature towards zero by $3.0^{\circ}$. Freezing of DOX formulation has to be carried out at temperatures below $-30.0^{\circ}$ and sublimation up to $-5.0^{\circ}$, for its accurate and flawless lyophilization. The lyophilized products obtained with the new lyophilization cycle complied with quality specifications, achieving a $35 \%$ reduction in the overall cycle duration.

\section{TABLE 1: CRITICAL STAGES DURING FREEZE-DRYING OF STUDIED BY LYOSTAT2}

\begin{tabular}{lcccc}
\hline \multirow{2}{*}{ Picture No. } & \multicolumn{2}{c}{ Placebo formulation } & \multicolumn{2}{c}{ DOX formulation } \\
\cline { 2 - 4 } & Temperature & Lyophilizate status & Temperature & Lyophilizate status \\
\hline 1 & $-34.2^{\circ}$ & No collapse & $-34.2^{\circ}$ & No collapse \\
2 & $-33.5^{\circ}$ & No collapse & $-33.5^{\circ}$ & No collapse \\
3 & $-30.9^{\circ}$ & No collapse & $-30.9^{\circ}$ & No collapse \\
4 & $-29.2^{\circ}$ & No collapse & $-29.2^{\circ}$ & No collapse \\
5 & $-25.1^{\circ}$ & No collapse & $-25.1^{\circ}$ & No collapse \\
6 & $-23.6^{\circ}$ & Drying progressing & $-23.6^{\circ}$ & Drying progressing \\
7 & $-21.5^{\circ}$ & Drying progressing & $-21.5^{\circ}$ & Drying progressing \\
8 & $-19.7^{\circ}$ & Majority dried & $-19.7^{\circ}$ & Majority dried \\
9 & $-19.7^{\circ}$ & Completely dried & $-19.7^{\circ}$ & Completely dried \\
\hline
\end{tabular}

TABLE 2: CRITICAL PARAMETERS OF THE LYOPHILIZATION PROCEDURE OF DOX FORMULATION

\begin{tabular}{|c|c|c|c|c|c|c|c|c|}
\hline \multirow{3}{*}{$\begin{array}{l}\text { Lyophilization } \\
\text { process }\end{array}$} & \multirow[b]{3}{*}{ Formulation } & \multicolumn{7}{|c|}{ Stages and parameters } \\
\hline & & \multicolumn{2}{|c|}{ Freezing } & \multicolumn{3}{|c|}{ Primary drying } & \multicolumn{2}{|c|}{ Secondary drying } \\
\hline & & $\begin{array}{c}\text { Freezing } \\
\text { temperature } \\
\left({ }^{\circ}\right)\end{array}$ & $\begin{array}{l}\text { Stabilization } \\
\text { time }(\mathrm{h})\end{array}$ & $\begin{array}{c}\text { Shelf } \\
\text { temperature } \\
\left({ }^{\circ}\right)\end{array}$ & $\begin{array}{l}\text { Pressure } \\
\text { (mBar) }\end{array}$ & $\begin{array}{c}\text { Safety } \\
\text { temperature } \\
\left({ }^{\circ}\right)\end{array}$ & $\begin{array}{c}\text { Shelf } \\
\text { temperature } \\
\left({ }^{\circ}\right)\end{array}$ & $\begin{array}{c}\text { Pressure } \\
\text { (mBar) }\end{array}$ \\
\hline Previous & DOX & -60 & 4 & -45 & 0.050 & -45 & 30 & $<0.050$ \\
\hline New & Placebo & -30 & 1 & -35 & 0.180 & -25 & 35 & 0.050 \\
\hline New & DOX & -30 & 1 & -35 & 0.160 & -25 & 35 & 0.050 \\
\hline \multicolumn{2}{|c|}{ Difference } & 30 & 1 & 10 & 0.11 & 20 & 5 & - \\
\hline
\end{tabular}

DOX: Doxorubicin hydrochloride

TABLE 3: CYCLE TIMES OF THE LYOPHILIZATION PROCESS OF THE DOX AT LABORATORY SCALE

\begin{tabular}{|c|c|c|c|c|}
\hline \multirow{2}{*}{ Lyophilization process } & \multicolumn{3}{|c|}{ Cycle times (h) } & \multirow{2}{*}{ Whole cycle $(\mathrm{h})$} \\
\hline & Freezing & Primary drying & Secondary drying & \\
\hline Previous process & 4 & 12 & 8 & 24 \\
\hline New process & 2.5 & 8 & 4 & 14.5 \\
\hline Difference & 1.5 & 4 & 4 & 9.5 \\
\hline
\end{tabular}




\section{Acknowledgements}

The authors thank Intas Pharmaceuticals for DOX as gift samples for this work. They also thank Dr. Shivajirao Kadam, Vice-Chancellor Bharati Vidyapeeth Deemed University, Pune and Dr. Vishwajit Kadam, Secretary, Bharati Vidyapeeth, Pune, for providing required facilities to carry out this research work.

\section{Conflicts of interest:}

There are no conflicts of interest.

\section{Financial support and sponsorship:}

Nil.

\section{REFERENCES}

1. Calabresi P, Chabner B. Antineoplastic agents. In: Gilman AG, Rall TW, Nies AS, Taylor P, editors. Goodman and Gilman's The Pharmacological Basis of Therapeutics. 8th ed. New York: Pergamon Press; 1990. p.1241.

2. Aristide V, Martin JW. Doxorubicin, In: Klaus F, editor. Analytical Profiles of Drug Substances. Cambridge, Massachusetts: Academic Press; 2005. p. 245-74.

3. Benvenuto JA, Anderson RW, Kerkof K. Stability and compatibility of antitumor agents in glass and plastic containers. Am J Hosp Pharm 1981;38:1914-8.

4. Beijnen JH, van der Houwen OAGJ, Underberg WJM. Aspects of the degradation kinetics of doxorubicin in aqueous solution. Int J Pharm 1986;32:123-31.

5. Tavoloni N, Guarino AM, Berk PD. Photolytic degradation of adriamycin. J Pharm Pharmacol 1980;32:860-2.

6. Pikal MJ. Freeze Drying. In: Swarbrick J, editor. Encyclopaedia of Pharmaceutical Technology, 3rd ed. Boca Raton, Florida: CRC Press; 2006. p. 1807-33.

7. Tang X, Pikal MJ. Design of freeze-drying processes for pharmaceuticals: practical advice. Pharm Res 2004;21:191-200.

8. Rey L, May JC. Freeze-Drying/Lyophilization of Pharmaceutical and Biological Products. New York: Marcel Dekker; 2004. p. 277-97.

9. Searles JA, Nail SL. Elements of quality design in development and scale-up of freeze dried parenterals. Biopharm Int 2008;21:44-52.

10. Smith G, Polygalov E, Arshad MS, Page T, Taylor J, Ermolina I. An impedance-based process analytical technology for monitoring the lyophilization process. Int $\mathrm{J}$ Pharm 2013;449:72-83.

11. Kasper JC, Winter G, Friess W. Recent advances and further challenges in lyophilization. Eur J Pharm Biopharm 2013;85:162-9.

12. Bosca S, Barresi AA, Fissore D. Fast freeze-drying cycle design and optimization using a PAT based on the measurement of product temperature. Eur J Pharm Biopharm 2013; 85:253-62.

13. Geidobler R, Winter G. Controlled ice nucleation in the field of freeze-drying: fundamentals and technology review. Eur J Pharm Biopharm 2013;85:214-22.

14. Shalaev EY, Johnson-Elton TD, Chang L, Pikal MJ. Thermophysical properties of pharmaceutically compatible buffers at sub-zero temperatures: implications for freezedrying. Pharm Res 2002;19:195-201.

15. Nail SL, Her LM, Proffitt CPB, Nail LL. An improved microscope stage for direct observation of freezing and freezedrying. Pharm Res 1994;11:1098-1100.

16. Hajare AA, More HN, Walekar PS, Hajare DA. Optimization of freeze drying cycle protocol using real time microscopy and integrated differential thermal analysis-electrical impedance. Res J Pharm Tech 2012;5:985-91.

17. Patel SM, Takayuki D, Pikal MJ. Determination of end point of primary drying in freeze-drying process control. AAPS PharmSciTech 2010;11:73-84.

18. Ma X, Wang DQ, Bouffard R, MacKenzie A. Characterization of murine monoclonal antibody to tumor necrosis factor (TNF$\mathrm{MAb}$ ) formulation for freeze-drying cycle development. Pharm Res 2001;18:196-202.

19. http://www.biopharma.co.uk/intelligent-freeze-drying/ products/lyotherm2-dta-impedance/.

20. Pikal MJ, Tang X, Nail SL. Automated process control using manometric temperature measurement. United States Patent US: 6,971,187 B1; 2005.

21. Rambhatla S, Ramot R, Bhugra C, Pikal MJ. Heat and mass transfer scale-up issues during freeze drying: II. Control and characterization of the degree of supercooling. AAPS PharmSciTech 2004;5:e58.

22. Tang X, Pikal MJ. Design of freeze-drying processes for pharmaceuticals: practical advice. Pharm Res 2004;21:191200.

23. Hajare AA, More HN, Pisal SS. Stabilisation of doxorubicin hydrochloride using sugar-phosphate glass composites prepared by vacuum foam drying. Eur J Parenteral Pharm Sci 2010;15:18-25.

24. Milton N, Pikal MJ, Roy ML, Nail SL. Evaluation of manometric temperature measurement as a method of monitoring product temperature during lyophilization. PDA J Pharm Sci Tech 1997;51:7-16.

25. Meister E, Gieseler H. Freeze-dry microscopy of protein/ sugar mixtures: drying behavior, interpretation of collapse temperatures and a comparison to corresponding glass transition data. J Pharm Sci 2009;98:3072-87.

26. Schersch KB. Effect of collapse on pharmaceutical protein lyophilizate [Ph.D. Thesis]. Munich, Germany: LudwigMaximilians University; 2009.

27. Rutzinger S. Freeze Drying Microscopy: Introduction and Relevance for Process Design. Erlangen: University of Erlangen-Numberg; 2009. Biotecnología Aplicada 2014; 32:1221-5.

28. Kasper JC, Winter G, Friess W. Recent advances and further challenges in lyophilization. Eur J Pharm Biopharm 2013; 85:162-9.

29. Arién S, Gerardo RH. Design of the lyophilization process of a L-leucyl-L-alanine dipeptide formulation based on its thermal properties. Biotecnología Aplicada 2014;32:1221-5.

30. Hajare AA, More HN, Pisal SS. Vacuum foam dried sugarphosphate amorphous mixturesfor stabilization of doxorubicin hydrochloride. Lat Am J Pharm2010:29;738-46. 